\title{
Avaliação de uma intervenção no treino assertivo e resolução de problemas em universitários
}

\author{
Camila Cristina Sancher. Dotta* \\ Francine Nathalie Quelur** \\ Vanessa Nunes de Soura***
}

\begin{abstract}
Resumo
A entrada em uma universidade pode ter como consequência dificuldades de ajustamento a novas demandas. Por isso, estabelecer novas relações interpessoais nesse contexto é de extrema importância. As habilidades sociais auxiliam o indivíduo a lidar de maneira adequada com as demandas das situações interpessoais. O treinamento assertivo e de resolução de problemas são componentes do treinamento em habilidades sociais. O objetivo do presente estudo foi verificar se o treinamento assertivo e de resolução de problemas contribuiriam para a diminuição dos níveis de ansiedade de estudantes universitários após uma intervenção, assim como a melhora no repertório de habilidades sociais. Para isso, foram avaliados seis estudantes de Psicologia, idade média de $22(D P=2,4)$ anos, sendo 4 mulheres e 2 homens, que responderam ao Inventário de Ansiedade de Beck e ao Inventário de Habilidades Sociais em três momentos: antes, após e depois de 01 mês de uma intervenção em grupo. De acordo com os resultados, o treinamento assertivo e de resolução de problemas propiciaram ganhos nas habilidades sociais gerais. No entanto, no que diz respeito aos resultados de ansiedade, não houve diferença estatisticamente significativa após a intervenção. Em estudos futuros seria importante avaliar se uma intervenção a longo prazo teria influência na ansiedade. Palavras-chave: Ansiedade, Treinamento de Habilidades Sociais, Intervenção.
\end{abstract}

\section{Assessment of an intervention in assertive training and solving problems in university students}

\begin{abstract}
Start into a university can lead to difficulties in adjusting to new demands. Therefore, establishing new interpersonal relationships in this context is extremely important. Social skills help the individual to deal adequately with the demands of interpersonal situations. Assertive and problem-solving training are components of social skills training. The aim of the present study was to verify if assertive and problem-solving training would contribute to the reduction of anxiety levels of university students after an intervention, as well as the improvement in the repertoire of social skills. For that, six Psychology students, mean age of $22(\mathrm{SD}=2.4)$ years, being 4 women and 2 men who responded to the Beck Anxiety Inventory and Social Skills Inventory in three moments: before, after and after 01 month of a group intervention. According to the results, assertive and problem-solving training provided gains in relation to general social skills. However, regarding the results of anxiety, there was no statistically significant difference after the intervention. In future studies it would be important to assess whether long-term intervention would influence anxiety. Key-words: Anxiety, Training of social skills, Intervention.
\end{abstract}

\footnotetext{
* Psicóloga pelo Centro Universitario Central Paulist - UNICEP e Mestranda em Enfermagem na UFSCar.

** Psicóloga, Mestre e Doutora em Psicologia pela UFSCar.

***Psicóloga, Mestre e Doutora pela UFSCar. Docente do curso de Psicologia da UNICEP
} 


\section{Introdução}

A entrada em uma universidade pode trazer dificuldades de interação e ajustamento às novas demandas, e por isso, estabelecer novas relações interpessoais dentro desse novo contexto é importante (Ribeiro \& Bolsoni-Silva, 2011). Nessa nova fase de vida, muitas situações podem contribuir para o aumento da ansiedade, como por exemplo, falar em público na apresentação de um seminário, expressar opiniões nas aulas, dar recados aos colegas, reivindicar com o professor notas e avaliações, trabalhar em grupo, ouvir, concordar/discordar, lidar com críticas justas e injustas, negociar, argumentar, perguntar, responder perguntas, entre outras (Del Prette \& Del Prette, 2017). De acordo com Santos (2014), estudantes universitários tendem a ser mais ansiosos, pois passam por uma fase de grandes transformações neurológicas, psicológicas e fisiológicas. Além disso, são expostos a uma grande carga de estresse decorrente de longas horas de estudos e cobranças familiares, pessoais e de professores.

A ansiedade é caracterizada por um estado subjetivo de apreensão ou tensão relacionado à sensação de que algo está para acontecer. É um sentimento avaliado como subjetivamente desagradável, que está acompanhado por alterações fisiológicas e comportamentais (Brandão, 2012). Quando a ansiedade se torna extremamente intensa e desconfortável, ela compromete a qualidade de vida do indivíduo, configurando uma psicopatologia, ou seja, um transtorno de ansiedade. Esse sentimento está ligado a um perigo incerto e potencial, a algo que pode vir a acontecer, mas que não necessariamente acontecerá. Assim, a experiência "estar ansioso", ou seja, apresentar algum sintoma de ansiedade é resultado da percepção ou intepretação de um determinado estímulo como ameaçador, tanto no âmbito físico quanto psicológico, o que pode gerar algumas mudanças no organismo, como também respostas comportamentais de fuga ou esquiva (Andretta \& Oliveira, 2011).

Padovani, Neufeld, Maltoni, Leopoldo, Souza e Cavalcanti(2014) buscaram identificar indicadores de bem- estar psicológico e vulnerabilidade em estudantes universitários, foram selecionados 3.587 estudantes (ambos os gêneros) de diversos cursos de seis instituições de ensino superior. Os instrumentos utilizados na pesquisa foram Inventário de Sintomas de Stress de Lipp, Inventário de Ansiedade Traço-Estado (IDATE), Inventário de ansiedade Beck (BAI) e Inventário de Depressão Beck
BDI. Com relação aos sintomas de ansiedade obtidos a partir do IDATE, Padovani et al. (2014), obtiveram como resultado de seu estudo a prevalência de sintomas de ansiedade em 13,54\% dos universitários, sendo que os resultados para o BAI mostraram a prevalência de sintomas nos níveis moderados e grave.

A literatura mostra que um indivíduo extremamente ansioso tem uma maior probabilidade de possuir um baixo repertório de habilidades sociais (Caballo, 2012). Nesse sentido, o Treinamento de Habilidades Sociais (THS) se encontra entre as técnicas mais utilizadas para o tratamento de problemas psicológicos pois está correlacionada a melhoria da afetividade interpessoal, e melhoria geral da qualidade de vida (Caballo, 2012). Ou seja, ser assertivo pode contribuir para a diminuição da ansiedade, uma vez que a assertividade (uma classe de habilidades sociais) é concebida como a expressão dos próprios sentimentos como uma necessidade independente dos sentimentos alheios, desde que esta não viole os direitos dos outros (Del Prette \& Del Prette, 2017). Neste sentido, ser assertivo pode fazer com que o indivíduo consiga se expressar de forma adequada, o que aumenta a probabilidade de atingir seus objetivos e consequentemente diminui a probabilidade de se sentir ansioso futuramente, pois sabe que se sairá bem ao se expressar ou se comportar. Outro conceito também foco deste trabalho é a capacidade de resolução de problemas, este processo diz respeito à tomada de decisão, ao controle emocional e à mediação de conflitos (Del Prette \& Del Prette, 2013).

Considera-se que a ansiedade e as respostas assertivas em conjunto com a capacidade de resolução de problemas são processos que atuam em sentidos opostos, pois de um lado a ansiedade pode inibir as iniciativas de interação (levando à esquiva ou à fuga dos contatos sociais) e de outro lado, a aquisição de respostas consideradas assertivas sendo emitidas em determinadas situações-problema, pode reduzir a ansiedade, já que o indivíduo consegue se expor em situações e momentos que antes não conseguiria (Bandeira, Quaglia, Bachetti, Ferreira, \& Souza, 2005). Um estudo realizado por Bandeira, Quaglia, Bachetti, Ferreira e Souza (2005), com 135 estudantes universitários, ambos os sexos, mostrou que quando o indivíduo se encontrava em situações de desempenho de papéis, os sujeitos com maior grau de ansiedade apresentavam um desempenho interpessoal inferior em termos de comportamentos assertivos verbais 
e não-verbais e em situações de alto ou baixo nível de avaliação por outras pessoas e constataram também que pessoas ansiosas foram avaliadas como menos assertivas e com mais cognições associadas a reações negativas. Por isso, a aquisição de respostas assertivas pode ajudar na redução da ansiedade.

No treinamento em resolução de problemas, ensina-se o indivíduo a perceber corretamente como executar alguns comportamentos considerados aceitáveis. A partir disso, é fornecida ao paciente a possibilidade de autonomia no que diz respeito às suas decisões facilitando a escolha da melhor opção para a resolução do problema (Del Prette \& Del Prette, 2013). Por isso, quando o indivíduo adquire um repertório para a resolução de problemas (em que soluções eficazes são aquelas respostas de enfrentamento que não só conseguem os objetivos desejados, mas que ao mesmo tempo maximizam consequências positivas e minimizam outras negativas), como o indivíduo acaba sendo estimulado/ exposto a solucionar de fato os problemas. Ensinam-se habilidades que permitam aos indivíduos vê-las de modo mais eficaz com problemas futuros, a fim de evitar ansiedade, ou manifestação de emoções negativas (Nezu \& Nezu, 2007).

Com relação às habilidades sociais, estas se referem a diferentes classes de comportamentos sociais no repertório de um determinado indivíduo que servem para que o mesmo possa lidar de maneira adequada com as demandas das situações interpessoais, entre elas, o manejo da ansiedade (Del Prette \& Del Prette, 2017). O treinamento de habilidades sociais, que engloba classes de habilidades como a assertividade e a resolução de problemas, é essencial quando se remete ao indivíduo ansioso, pois a literatura mostra que um indivíduo extremamente ansioso possui uma alta probabilidade de ter também um baixo repertório de habilidades sociais (Del Prette \& Del Prette, 2013). Diante disso, o Treinamento de Habilidades Sociais se apresenta como uma alternativa, e depende dos resultados práticos e teóricos de sua aplicação, principalmente no que diz respeito à superação dos déficits e dificuldades interpessoais e também ao aumento de repertórios de comportamentos socialmente assertivos (Del Prette \& Del Prette, 2012).

O treino assertivo e de resolução de problemas são habilidades contempladas no Treinamento de Habilidades Sociais e que auxiliam o indivíduo a lidar com questões cotidianas, como por exemplo, situações que envolvem confronto de opiniões, tarefas de falar em público, situações de expressão de sentimentos e exercício de direitos, que normalmente provocam intenso desconforto em pessoas ansiosas (Del Prette \& Del Prette, 2013). Buscando entender um pouco mais sobre as habilidades sociais no contexto acadêmico, Del Prette, Barreto, Bandeira, Salvaña, Ulian, Carneiro, Falconi e Villa (2004) verificaram quais classes de habilidades sociais 564 estudantes de Psicologia tinham em seu repertório comportamental. Para tal, utilizaram o Inventário Habilidades Sociais (IHS). O trabalho apontou que os estudantes apresentaram um repertório mais desenvolvido nas classes enfrentamento e autoafirmação de risco (que está dentro da assertividade), na conversação e desenvoltura social e na auto exposição a desconhecidos e situações novas. Por outro lado, apresentaram um menor repertório nas classes de habilidades relacionadas à autoafirmação na expressão de sentimento positivos e ao autocontrole da agressividade. Os autores afirmaram que essa menor prevalência pode ter ocorrido em decorrência de um ajustamento do estudante ao estereótipo do psicólogo como um profissional mais contido e controlado (que não expressa sentimentos de afeto, o que poderia ser reforçado ao longo da graduação), ou ainda a déficits de habilidades sociais nessa área, que o curso não estaria suprindo.

Com o objetivo de também promover a aquisição de habilidades sociais, emocionais e comportamentais para o manejo da ansiedade em situações de exposição ao público e em conjunto com o efeito das mesmas na ansiedade, Barreto, Bandeira, Carneiro, Del Prette, Del Prette, Falcone, Ulian e Villa (2004) investigaram os efeitos, a curto e a longo prazo, do treinamento de habilidades sociais em 34 universitários. Para tal, utilizaram o Inventário Habilidades Sociais (IHS) e o Inventário de Ansiedade Traço-Estado (IDATE) para fazer 3 (três) comparações (pré-teste, pós-teste e follow up). O estudo demonstrou, a partir da análise do IHS, que 67,6\% dos participantes tiveram um aumento confiável no percentil de habilidades sociais após as intervenções. Demonstrou, também, a partir da análise do IDATE, que antes das intervenções, os indivíduos apresentavam altos níveis de ansiedade e que após as intervenções, os universitários passaram a apresentar níveis de leve a moderado de ansiedade, que se mantiveram no follow up.

No estudo realizado pelos autores Silva, Leme, Lima e Correia (2009), com 18 estudantes universitários, 
avaliou a efetividade de um treinamento de habilidades sociais por meio de medidas de avaliação pré e pós-teste, utilizando o Inventário de Habilidades Sociais (IHS) nos dois momentos de avaliações. O programa teve vinte encontros semanais em grupo. A pesquisa apontou que os estudantes, após passarem pelo treinamento, obtiveram ganhos estatisticamente significativos nos seguintes fatores do IHS: fator F1 (manter conversa com desconhecidos, cobrar dívida de amigos, apresentar-se a outra pessoa, abordar para relação sexual e discordar do grupo), F3 (abordar autoridade e manter conversação) e F4 (falar a público desconhecido, fazer pergunta e/ou pedir favores a desconhecidos). Nesse estudo, o grupo não apresentou melhoras estatisticamente significativas em F2 (agradecer elogios, elogiar familiares, participar de conversação, expressar sentimento positivo, defender outrem em grupo, elogiar outrem e expressar sentimento positivo) nem em F5 (lidar com críticas dos pais, lidar com chacotas, cumprimentar desconhecidos, pedir mudança de conduta, lidar com críticas justas, recusar pedido abusivo e interromper a fala do outro). De acordo com os autores do trabalho, uma explicação para o fato de não se conseguir mudanças em F5 se dá pelo fato de alguns estudantes não estarem mais morando com os pais ou familiares (já que três perguntas são feitas de acordo com a aplicação do IHS, tais como: lidar com a crítica dos pais, lidar com chacotas e cumprimentar desconhecidos). Ou seja, nesse caso, os participantes não adquiriram habilidades relacionadas a esse princípio em função do ambiente não propiciar condições para o uso de tais habilidades. Outra hipótese apresentada pelos autores é referente ao tempo da intervenção, que pode ter sido insuficiente para captar tais mudanças (Silvia, Leme, Lima \& Correia, 2009).

Ferreira, Oliveira e Vandenbergh (2014) investigaram os efeitos, a curto e longo prazo, do treinamento de habilidades sociais em um grupo de universitários. Utilizaram o Inventário Habilidades Sociais (IHS) e o de ansiedade Inventário de Ansiedade Traço (IDATE). Foram feitas 3 (três) comparações (pré-teste, pós-teste e follow up). No estudo, os fatores de enfrentamento não foram comparados separadamente, mas os resultados, obtidos como um todo foi significativo com relação ao ganho de Habilidades Sociais. Com relação a ansiedade dos participantes, ocorreu uma diminuição da mesma e estes resultados foram mantidos a longo prazo. Os mesmos autores ainda demonstraram que melhora nos escores de habilidades sociais e de ansiedade se mantiveram em longo prazo, com o período de follow up.

Nesta perspectiva, acredita-se que indivíduos ansiosos podem se beneficiar do treinamento assertivo e de resolução de problemas já que a aquisição de tais habilidades pode contribuir para a diminuição da intensidade das manifestações ansiosas, haja vista que as situações que outrora eram consideradas aversivas podem deixar de possuir caráter ameaçador. Dado o exposto, o presente estudo focou na assertividade e na resolução de problemas, que são duas classes de habilidades sociais. Tanto a assertividade, quanto a habilidade de resolução de problemas podem ser aprendidas, logo, podem também ser treinadas (Braz, Cômodo, Del Prette, Del Prette, \& Fontaine, 2013). Estas habilidades foram contempladas com o objetivo do presente estudo de verificar se o treino assertivo e o treino de resolução de problemas contribuiriam para a diminuição dos níveis de ansiedade de estudantes universitários após uma intervenção, assim como a melhora no repertório de habilidades sociais.

\section{Método}

\section{Participantes}

O presente estudo iniciou-se com 16 participantes, com idade média de $22(D P=2,4)$ anos, sendo $4 \mathrm{mu}$ lheres e 2 homens, todos estudantes universitários do curso de Psicologia de uma instituição do interior de São Paulo. Por ser um estudo de intervenção, a desistência de participantes durante o estudo foi inevitável. Dez participantes relataram desistir por terem dificuldade de chegar no horário estipulado. Portanto, os dados analisados no presente estudo serão dos seis participantes que completaram todas as etapas da intervenção. Desses 6 participantes, a idade média foi de $22(D P=2,4)$ anos, sendo 4 do sexo feminino e 2 do sexo masculino. Todos eram universitários do primeiro ano do curso de Psicologia de uma faculdade particular localizada no interior do estado de São Paulo. Os dados foram coletados no Serviço Escola de Psicologia, em salas utilizadas para atendimento psicológico.

\section{Instrumentos}

Inventário de Ansiedade de Beck (BAI) (Beck, 2001). O BAI se refere a uma escala de auto relato, que mede a intensidade de sintomas de ansiedade e foi validada para 
o Brasil por Cunha (2001). O Inventário é constituído por 21 itens, que constituem "afirmações descritivas de sintomas de ansiedade" e devem ser avaliados pelo sujeito com referência a si mesmo, em uma escala de quatro pontos. Estes refletem níveis de gravidade crescente de cada sintoma: (1) "Absolutamente não"; (2) "Levemente: não me incomodou muito"; (3) "Moderadamente: foi muito desagradável, mas pude suportar"; (4) "Gravemente: dificilmente pude suportar" (Cunha, 2011). Os níveis dos escores do BAI da versão em português podem assumir escores de zero (0) a dez (10), o que sugere nível mínimo de ansiedade, de onze (11) a dezenove (19), nível leve, de vinte (20) a trinta (30), nível moderado e de trinta e um (31) a sessenta e três (63), nível grave de ansiedade (Cunha, 2001).

Inventário de Habilidades Sociais (IHS) - Del Prette e Del Prette (2001). O teste foi concebido e validado por Del Prette e Del Prette (2000) com o intuito de avaliar o repertório interpessoal. Os itens do instrumento englobam vários contextos tais como público, privado ou indefinido; diversos tipos de interlocutores (família, desconhecido, autoridade) e variadas demandas interpessoais como reações e comportamentos desejáveis e indesejáveis do interlocutor e emissões que não dependiam da ação explícita do interlocutor. O Inventário de Habilidades Sociais (IHS) contém 42 itens, cada um apresentando uma ação ou sentimento diante de uma determinada ação social. $\mathrm{O}$ indivíduo precisa indicar a frequência com que age ou sente o que foi descrito em cada item. Para isso, é necessário preencher uma folha de respostas situada ao final dos itens, onde consta a legenda de uma escala tipo Likert, com cinco pontos: A: nunca ou raramente; B: com pouca frequência; C: com regular frequência; D: muito frequentemente; E: sempre ou quase sempre. Os fatores avaliados no IHS são: Fator 1: Enfrentamento e Autoafirmação com Risco; Fator 2: Autoafirmação e Expressão de Sentimentos Positivos; Fator 3: Conversação e Desenvoltura Social; Fator 4: Auto exposição a Desconhecidos e Situações Novas e Fator 5: Autocontrole da Agressividade.

\section{Procedimento}

Após a autorização do comitê de ética do Centro Universitário Central Paulista-UNICEP (Aprovação CEP-UNICEP, número: 32095614.6.0000.5380), os participantes foram convidados a participar da pesquisa na própria sala de aula. Após assinarem o Termo de Consentimento
Livre e Esclarecido, os participantes responderam ao BAI (pré-teste $-1^{a}$. aplicação) para constatação de indicativo ou não de ansiedade, bem como da severidade dos sintomas e em seguida, responderam ao IHS, o que permitiu a realização de um levantamento inicial do repertório de habilidades sociais dos mesmos.

Em seguida, o grupo foi submetido a 15 (quinze) sessões de intervenção, sendo 8 (oito) sessões de treinamento assertivo e 7 (sete) sessões de treinamento de resolução de problemas. $\mathrm{Na}$ semana posterior ao término das intervenções grupais, os participantes foram submetidos à $2^{a}$ aplicação do BAI e do IHS (pós teste). Em seguida, passado um período de 30 dias da última avaliação, o grupo foi novamente avaliado por meio do BAI e do IHS, o que resultou na $3^{a}$ aplicação dos testes (follow up).

\section{Análise de Dados}

Todos os dados foram codificados e interpretados segundo o Manual de Aplicação de cada um dos testes, e a partir disso, foi feita uma comparação entre as três avaliações por meio de análise estatística. Utilizou-se o "Teste F" com o objetivo de verificar se havia diferença estatisticamente significativa entre as variâncias das populações da amostra. Em seguida, os dados foram submetidos ao Teste T de Student para populações com variâncias distintas com o objetivo de comparar e verificar se houve alteração estatisticamente significativa nos resultados obtidos pela amostra com um índice de confiança menor ou igual a 0,05 . Em primeiro lugar foi comparado o resultado da aplicação dos testes IHS e BAI na pré intervenção (pré-teste $-1^{a}$. aplicação) com os resultados obtidos na pós intervenção (pós teste $2^{\mathrm{a}}$. aplicação); em seguida comparou-se os resultados obtidos na pós intervenção ( $3^{a}$ aplicação) com os resultados obtidos após o período de follow up. Além disso, calculou-se o tamanho do efeito por meio do D de Cohen (pontos para classificação do tamanho de efeito, os valores superiores ou iguais a 0,8 representam tamanho de efeito grande; entre 0,8 a 0,2 são considerados médios e inferiores a 0,2 pequenos) para as medidas que apresentaram diferença estatisticamente significativa, que é uma estimativa utilizada para indicar a significância prática, esse resultado fornece uma medida de diferença de grupos em termo de desvio padrão. 


\section{Resultados}

Tabela 1. Comparação das variáveis do IHS e BAI durante o pré e pós teste e follow up.

\begin{tabular}{|c|c|c|c|c|c|c|}
\hline ETAPA & $\mathbf{n}$ & FATORES & MÉDIA INICIAL & MÉDIA FINAL & p & D de Cohen \\
\hline & 6 & GERAIS & $40(27)$ & 68 & $0,04 *$ & $-0,56$ \\
\hline IHS COMPARAÇÃO & & FATOR 1 & 43 & 62 & 0,06 & \\
\hline \multirow[t]{5}{*}{ PRÉ-TESTE x PÓS TESTE } & & FATOR 2 & 35 & 58 & 1,88 & \\
\hline & & FATOR 3 & 35 & 70 & $0,04 *$ & $-0,56$ \\
\hline & & FATOR 4 & 31 & 64 & $0,02 *$ & $-0,54$ \\
\hline & & FATOR 5 & 45 & 51 & 0,31 & \\
\hline & 6 & GERAIS & $68(24)$ & 73 & 0,35 & \\
\hline IHS COMPARAÇÃO & & FATOR 1 & 62 & 56 & 0,32 & \\
\hline \multirow[t]{6}{*}{ PRÉ TESTE x FOLLOW UP } & & FATOR 2 & 58 & 61 & 0,43 & \\
\hline & & FATOR 3 & 70 & 75 & 0,38 & \\
\hline & & FATOR 4 & 64 & 45 & 0,13 & \\
\hline & & FATOR 5 & 51 & 58 & 0,3 & \\
\hline & & GERAIS & $40(20)$ & 73 & $0,01^{*}$ & $-0,63$ \\
\hline & & FATOR 1 & 46 & 56 & 0,15 & \\
\hline IHS COMPARAÇÃO & 6 & FATOR 2 & 35 & 61 & $0,03 *$ & $-0,48$ \\
\hline \multirow[t]{3}{*}{ PRÉ TESTE X FOLLOW UP } & & FATOR 3 & 35 & 75 & $0,03 *$ & $-0,64$ \\
\hline & & FATOR 4 & 31 & 45 & 0,19 & \\
\hline & & FATOR 5 & 45 & 58 & 0,14 & \\
\hline BAI COMPARAÇÃO & 6 & & & & & \\
\hline PRÉ TESTE x PÓS TESTE & & & $17,5(11)$ & 19,5 & 0,41 & \\
\hline BAI COMPARAÇÃO & 6 & & & & & \\
\hline PÓS TESTE x FOLLOW UP & & & $19,5(20)$ & 14 & 0,3 & \\
\hline BAI COMPARAÇÃO & 6 & & & & & \\
\hline PRÉ TESTE x FOLLOW UP & & & $17,5(14)$ & 14 & 0,32 & \\
\hline Legenda: $* \leq 0,05$ & & & & & & \\
\hline & & & & & & \\
\hline
\end{tabular}

\section{Habilidades Sociais}

Com relação ao escore geral do instrumento de habilidades sociais, a análise estatística demonstrou aumento estatisticamente significativo de 40 (pré-teste) para 68 (pós teste); com relação ao d de Cohen, de acordo com o coeficiente de correlação e interpretação, os participantes apresentaram resultado de $-0,56$ que resulta em uma magnitude grande. Na comparação entre pré-teste e follow up houve um aumento de 67 para 73; e na condição pré-teste e follow up o valor dos escores foi de 40 para 73; com relação ao d de Cohen, de acordo com o coeficiente de correlação e interpretação, os participantes apresentaram resultado de -0,63 que resulta em uma magnitude grande.
Para o fator (F1) enfrentamento e autoafirmação com risco, a análise dos dados demonstrou um aumento de 43 (pré-teste) para 62,16 (pós-teste) nas médias obtidas para o fator F1. Deve-se considerar, entretanto, uma tendência a aumento estatisticamente significativo nas médias para o fator F1 entre as condições pré e pós teste já que o valor de p obtido $(p=0,06)$ foi muito próximo do nível de significância de 5\%. No "follow up", os participantes apresentaram uma diminuição da média relacionada ao percentil das habilidades sociais de F1, pois a mesma foi de 62 (pré-teste) para 56 (follow up), no entanto essa diferença não foi estatisticamente significativa. Nota-se, portanto, que ocorreu uma tendência a aumento nos va- 
lores das médias relativas aos percentis das habilidades sociais de F1 após a intervenção e que esta tendência de aumento se manteve após o follow up.

No que diz respeito ao fator (F2) autoafirmação na expressão de sentimento positivo os participantes obtiveram aumento estatisticamente significativo $(p=0,05)$ na média de habilidades sociais de F2 após o teste, pois a mesma aumentou de 35 (pré-teste) para 58 (pós teste). Nota-se que no "follow up", os participantes apresentaram um aumento da média relacionada ao percentil das habilidades sociais de F2, pois a mesma foi de 58 (pós-teste) para 61 no follow up. Esta diferença nas médias do pós-teste e follow up não é, contudo, estatisticamente significativa, já que o valor de $p$ foi maior que $5 \%$ ou 0,05. Dessa forma, para o fator F2, a análise estatística demonstrou aumento nas médias relativas aos percentis obtidos após a intervenção. A análise dos dados demonstrou ainda que este aumento se manteve após o follow up.

Para o fator (F3) conversação e desenvoltura social os participantes obtiveram aumento estatisticamente significativo $(p \leq 0,05)$ na média de habilidades sociais de F3 após a intervenção, pois a mesma aumentou de 35 (pré-teste) para 70 (pós teste). Nota-se que no "follow up", os participantes apresentaram um aumento da média relacionada ao percentil das habilidades sociais de F3, pois a mesma foi de 70,25 para 75,75. Esta diferença nas médias das condições pós teste e follow up não foi estatisticamente significativa. Assim como ocorreu para o fator 2, a análise estatística demonstrou aumento nas médias relativas aos percentis obtidos para o fator 3 após o período de intervenção. A análise dos dados também demonstrou que este aumento se manteve após o follow up. Com relação ao d de Cohen, de acordo com o coeficiente de correlação e interpretação, os participantes apresentaram resultado de $-0,54$ que resulta em uma magnitude grande. Em relação ao fator (F4) auto exposição a desconhecidos e situações novas os participantes obtiveram aumento estatisticamente significativo $(p \leq 0,05)$ na média de habilidades sociais de F4 após a intervenção, pois a mesma aumentou de 31 (pré-teste) para 64 (pós teste). Nota-se que após o "follow up", os participantes apresentaram uma diminuição da média relacionada ao percentil das habilidades sociais de F4, pois a mesma foi de 64 (pós teste) para 45 (follow up). Esta diminuição, entretanto, não foi considerada estatisticamente significativa ( $p>0,05)$. A análise estatística demonstrou, portanto, aumento nas médias relativas aos percentis obtidos para o fator 4 após o período de intervenção, condição que se manteve após o follow up. Com relação ao d de Cohen, de acordo com o coeficiente de correlação e interpretação, os participantes apresentaram resultado de $-0,56$ que resulta em uma magnitude grande.

Para o fator (F5) autocontrole da agressividade os participantes obtiveram aumento na média de habilidades sociais de F1 após a intervenção, pois a mesma aumentou de 45 (pré-teste) para 51 (pós teste). Este aumento, entretanto, não foi considerado estatisticamente significativo. A análise estatística demonstrou que no "follow up", os participantes apresentaram aumento da média relacionada ao percentil das habilidades sociais de F5, pois a mesma foi de 51 (pós teste) para 58 (follow up). Este aumento, contudo, não foi considerado estatisticamente significativo, pois a análise dos resultados mostrou um valor de $p$ maior que 0,05 . No que diz respeito ao fator 5 , a análise estatística não apontou diferença estatisticamente significativa nas médias obtidas pelos participantes antes e após a intervenção. Este resultado se manteve após o follow up.

\section{Ansiedade}

A análise estatística mostrou aumento na média dos escores relacionados aos índices de ansiedade de 17,5 (ansiedade moderada - pré-teste) para 19,5 (ansiedade moderada - pós teste). A análise estatística não demonstrou, entretanto, aumento estatisticamente significativo com relação às médias, já que o valor de $p$ obtido foi maior que 0,05 . Com relação às médias dos escores do BAI obtidos pelos participantes no pré-teste e no "follow up", a análise estatística apontou uma diminuição na média dos escores relacionados aos índices de ansiedade de 19,5 (ansiedade moderada - pós-teste) para 14,0 (ansiedade moderada - follow up). Mas análise estatística não demonstrou, diminuição estatisticamente significativa com relação às médias, já que o valor de $\mathrm{p}$ obtido foi maior que 0,05 . Dessa forma, a análise dos dados demonstrou que não houve diferença estatisticamente significativa no que diz respeito aos índices de ansiedade obtidos antes e após a intervenção. Esta condição se manteve após o follow up.

\section{Discussão}

O objetivo do presente estudo foi verificar se o treinamento assertivo e de resolução de problemas contribuiriam para a diminuição dos níveis de ansiedade de estudantes universitários após uma intervenção, assim como a melhora no repertório de habilidades sociais. Percebe-se a partir disso que os resultados demonstraram que as intervenções feitas com o grupo foram efetivas em partes. Já que, a partir delas, foi possível que os participantes ampliassem seu repertório de habilidades 
sociais, mas não houve diminuição estatisticamente significativa na ansiedade. No que diz respeito às habilidades sociais, é importante ressaltar que esse repertório se manteve, ainda após passados 30 dias (follow up) do término das intervenções. Ou seja, os participantes "ganharam" melhores recursos interpessoais a partir das intervenções.

Os resultados encontrados nas avaliações pré e pós teste sugerem que o Treinamento de Habilidades Sociais (especificamente o treinamento assertivo e de resolução de problemas) foram eficazes em desenvolver um conjunto de comportamentos que os participantes não apresentavam antes, como, por exemplo, ser assertivo, lidar com críticas, expressar raiva, expressar sentimentos positivos, agradecer elogios, pedir favores a outras pessoas e recusar pedidos abusivos.

Ao analisar especificamente os resultados de cada fator do Inventário de Habilidades Sociais, notou-se que a intervenção promoveu ganhos estatisticamente significativos para as habilidades que compõem F2 (autoafirmação na expressão de sentimento positivo), F3(conversação e desenvoltura social) e F4 (auto exposição a desconhecidos e situações novas). Com relação ao F1(enfrentamento e autoafirmação com risco), o aumento nos percentis obtidos a partir da comparação entre a pré e a pós intervenção não foram considerados significativos, mas como o nível de significância estatística para este fator foi de 0,06\% (valor muito próximo do nível de significância de $0,05 \%$ ), considera-se uma forte tendência a aumento. Nota-se que o F5 foi o único em que não houve alteração estatisticamente relevante entre os valores obtidos nos percentis na pré e pós-intervenção. Acredita-se que não houve diferença nesse fator pois esta é uma habilidade que se expressa como uma característica interpessoal importante e, muitas vezes, reflete uma característica do indivíduo, conhecida como impulsividade (incompatível com a calma e o autocontrole avaliados nesse fator) (Awang, Kutty, Ahamad, 2014). O autocontrole da agressividade se dá nas situações que envolvem algum risco de consequências negativas, e se caracteriza por um tipo de enfrentamento que requer o autocontrole de sentimentos negativos despertados pela ação do outro e a expressão apropriada desses sentimentos (Del Prette \& Del Prette, 2011; 2017).

O treino assertivo realizado no presente estudo foi de extrema importância, pois se o indivíduo se comporta de uma maneira assertiva, pode alcançar recompensas sociais mais facilmente e assim obtém satisfação pessoal (Del Prette \& Del Prette, 2012). Sabe-se que um indivíduo nem sempre será na sua totalidade assertivo, visto que, em uma determinada situação, este pode ser assertivo e em outras situações pode apresentar comportamentos passivos ou agressivos (Del Prette \& Del Prette, 2012; 2017), no entanto, uma vez que o indivíduo adquire essas habilidades em seu repertório comportamental a probabilidade de vir a emitir comportamentos socialmente habilidosos aumenta e é nessa direção que estudos de intervenção como este são importantes, uma vez que as habilidades sociais podem ser treinadas visando uma melhor qualidade de vida geral em diferentes populações (Del Prette \& Del Prette, 2017).

O treinamento especificamente de resolução de problemas, dentro das habilidades sociais, também é uma ferramenta importante, uma vez que os problemas são situações específicas da vida e que exigem respostas para o funcionamento adaptativo, de maneira que, ao encontrá-los, o indivíduo esteja apto a enfrentá-los e até mudar sua forma de se comportar diante de demandas interpessoais diferentes a depender de cada contexto (Del Prette \& Del Prette, 2013; Pinto, Barham, \& Del Prette, 2016). Deste modo, é necessário avaliar a natureza da situação problema e identificar um conjunto de objetivos ou metas realistas que possibilitem a resolução do mesmo. Com isso minimiza-se o grau de influência negativa para as tentativas futuras de enfrentamento, e treina-se habilidades que permitam que os indivíduos possam vê-las de modo mais eficaz com relação a problemas futuros, a fim de evitar desconfortos psicológicos (Santos, 2014). Nesse sentido, percebe-se que os ganhos dos participantes, no presente estudo, indicaram que os indivíduos adquiriram habilidades para lidar com algumas situações que antes eram vistas como ruins ou ameaçadoras, tais como lidar com críticas (muitas vezes injustas), fazer perguntas a pessoas desconhecidas, discordar de alguém, falar em público, manter conversação com desconhecidos, agradecer elogios, expressar sentimento positivo, pedir favores a colegas, reagir a um elogio, recusar pedidos abusivos, pedir mudança de comportamento e expressar desagrado a alguém.

Com relação à média dos escores de ansiedade, não houve diferença estatisticamente significativa após a intervenção, pois o grupo obteve escore 17,5 (ansiedade moderada) no pré-teste, 19,0 (ansiedade moderada) no pós-teste e um escore médio de 14 pontos (ansiedade moderada) no follow up, o que indica que não houve alteração nos níveis de ansiedade do grupo. A literatura (Pureza, Rusch, Wagner \& Oliveira, 2012) aponta que pode ser difícil modificar índices de ansiedade em grupos, já que de um modo geral, a psicoterapia grupal gera certo nível de ansiedade na maioria dos participantes e, somente após 
algum tempo, os mesmos vão estabelecendo uma relação de aceitação e confiança, que pode ter como consequência a diminuição da ansiedade, mas em longo prazo(Pureza, Rusch, Wagner, \& Oliveira, 2012). No presente estudo, parece que quinze sessões de intervenção foram incipientes na melhora dos índices de ansiedade, talvez se fosse um estudo de longo prazo (um ano por exemplo), os resultados seriam mais representativos.

Nota-se que a maioria dos estudos sobre ansiedade não envolvem intervenções para os participantes do estudo e fazem apenas levantamento de dados que acabam por resultar em dados quantitativos, o que gera uma dificuldade na comparação e discussão dos resultados deste trabalho com os dados da literatura. Uma outra dificuldade encontrada no presente estudo ocorreu em função da escassez de trabalhos que relacionassem treinamento de habilidades sociais e ansiedade. No entanto, essa escassez serviu como agente motivador para a execução deste trabalho. Outra limitação que dificulta a comparação dos resultados deste estudo com outros trabalhos já realizados sobre esse tema se refere ao tamanho reduzido desta amostra ao final da intervenção, uma vez que a maioria dos participantes desistiu de participar das intervenções ao longo do processo.

Por fim, a grande dificuldade, em relação à condução da pesquisa, deu-se em função da baixa adesão no seu início e desistência durante o seu percurso, devido ao fato de muitos participantes trabalharem durante o dia e apresentarem dificuldades de disponibilidade de horários livres. O estudo iniciou-se com 16 participantes, com idade média de $22(D P=2,4)$ anos, sendo quatro mulheres e dois homens, todos estudantes universitários do curso de Psicologia de uma instituição do interior de São Paulo. Por ser um estudo de intervenção, a desistência de participantes durante o estudo foi inevitável. Dez participantes relataram desistir por terem dificuldade de chegar no horário estipulado. Como ocorreu uma diminuição no número de participantes, as 20 (vinte) sessões de intervenção planejadas no início foram reduzidas para 15 (quinze), com o intuito de evitar mais perda de participantes do grupo.

A despeito dessas questões, é importante ressaltar que os participantes adquiriram habilidades para lidar com situações que antes eram vistas como ruins ou ameaçadoras. Vale ressaltar que ainda são necessários novos estudos científicos sobre o tema, com um número maior de participantes na pesquisa, um tempo mais longo de intervenção e também se faz necessário a pesquisa em outras populações alvo.

\section{Conclusão}

De maneira geral, o estudo apontou que as sessões de intervenção resultaram em um aumento dos escores de habilidades sociais. Os efeitos foram estatisticamente significativos e se mantiveram após 30 dias do término das intervenções. Foi possível notar que as intervenções (o treinamento assertivo e o de resolução de problemas) trouxeram efeitos positivos e ganhos significativos para os indivíduos, pois os mesmos, a partir das vivências cujo objetivo era proporcionar desempenhos e experiências interpessoais que articulassem demandas cognitivas, emocionais e comportamentais, mudaram significativamente seus percentis de habilidades sociais no geral e nos fatores enfrentamento autoafirmação na expressão de sentimentos positivos, conversação e desenvoltura social. A intervenção não foi capaz de modificar os índices de ansiedade. Ressalta-se que a ansiedade é uma condição complexa e multifatorial, cujo manejo a partir de intervenções grupais demanda tempo para que os participantes se familiarizem uns com os outros e adquiram confiança para se expor a contingências que envolvem aquisição de competências. A literatura aponta que pessoas que tem um maior repertório de habilidades apresentam menores índices de ansiedade. Nesse sentindo, espera-se que a longo prazo, com o desenvolvido das habilidades que os participantes aprenderam neste estudo e a generalização para outros contextos, que os níveis de ansiedade apresentados pelos menos diminuía, contribuindo para a melhora da sua qualidade vida e consequentemente de sua vida acadêmica.

\section{Referências}

Awang, M., M., Kutty, F., M., \& Ahmad, A., R. (2014). Perceived Social Support and Well Being: Fisrt-Year Student Experience in University. International Education Studies (Canada), 13(7), 261-269. Doi: :10.5539/ies.v7n13p261

Bandeira, M., Quaglia, M. A. C., Bachetti, L. D. S., Ferreira, T. L., \& Souza, G. G. (2005). Assertive behavior and its relation to anxiety, locus of control and self esteem in undergraduate students. Estudos de Psicologia (Campinas), 22(2), 111-121. https://doi.org/10.1590/S0103-166X2005000200001

Barreto, M.C.M., Bandeira, M., Carneiro, E.G., Del Prette, Z.A.P., Del Prette, A., Falcone, E.M.O., Ulian, A.L.A.O., Villa, M.B. Habilidades Sociais de Estudantes de Psicologia: Um Estudo Multicêntrico. Psicologia: Reflexão e Crítica, 2004, 17(3), pp.341-350 Recuperado de http://www.scielo.br/pdf/prc/ v17n3/a07v17n3.pdf

Brandão, M. L., \& Graeff, F. G. (2012) Ansiedade. Em M. L. Brandão. Fundamentos da Psicofarmacologia (pp. 67-155). São Paulo, SP Atheneu.

Braz, A. C., Cômodo, C. N., Del Prette, Z. A. P., Del Prette, A., \& Fontaine, A. M. G. (2013). Habilidades sociales e intergeneracionalidad en las relaciones familiares. Apuntes de Psicologia, 31(1), 77-84. Recuperado de http:/ / www. apuntesdepsicologia.es/index.php/ revista/article/view/305

Caballo, V.E. (2012) Manual de Avaliação e Treinamento das Habilidades Sociais. Habilidades sociais: quadro teórico. São Paulo, Santos.

Cunha, J.A. (2011) Manual da versão em português das escalas de Beck. São Paulo, Casa do Psicólogo. 
Del Prette, Z. A. P., \& Del Prette. (2001). Inventário de Habilidades Sociais (IHS -Del Prette): Manual de aplicação, apuração e interpretação. São Paulo, SP. Casa do Psicólogo

Del Prette, A., \& Del Prette, Z.A.P. (2003) No contexto da travessia para o ambiente de trabalho: treinamento de habilidades sociais com universitários. Estudos de Psicologia, 8(3), 413-420. bttp://dx.doi.org/10.1590/S1413-294X2003000300008

Del Prette, A., Del Prette, Z. A. P., Barreto, M. C. M., Bandeira, M., Salvaña, M. R. R., Ulian, A. L. A. O., Carneiro, E. G., Falconi, E. M., \& Villa, M. B. (2004) Habilidades Sociais em estudantes de psicologia: um estudo multicêntrico. Psicologia: Reflexão e Crítica, 17(3), 341-350. Recuperado de http:/ / www.scielo.br/pdf/prc/v17n3/a07v17n3.pdf

Del Prette, A., Del Prette, Z.A.P. (2011) Psicologia das Habilidades Sociais: terapia, educação e trabalho. Desenvolvimento das habilidades sociais (pp 17-22) Petrópolis, Vozes.

Del Prette, A., \& Del Prette, Z.A.P. (2012) Psicologia das Habilidades Sociais: terapia, educação e trabalho. Desenvolvimento das habilidades sociais (pp 17-19). Petrópolis, Vozes.

Del Prette, A. \& Del Prette, Z.A.P. (2001). Psicologia das Habilidades Sociais - Terapia e Educação (pp 25-35). Petrópolis. Vozes Editora.

Del Prette, A., \& Del Prette, Z.A.P. (2017) Competência Social e Habilidades Sociais: Manual teórico-prático (pp 17-25). Petrópolis, Vozes.

Ferreira, V. S., Oliveira, M. A., \& Vandenberghe, L. (2014). Efeitos a curto e longo prazo de um grupo de desenvolvimento de habilidades sociais para universitários. Psicologia: Teoria e Pesquisa, 30(1),73-81. https://dx.doi. org/10.1590/S0102-37722014000100009

Nezu, A. M., \& Nezu, C. M. Manual de Técnicas de Terapia de Modificação Comportamental. In Treinamento em Solução de Problemas(pg 476-477). São Paulo, 2007.
Padovani, R. C., Neufeld, C. B., Maltoni, J. B., Leopoldo N. F., Souza, W. F., Cavalcanti, H. A. F, \& Lameu, J. N., (2014). Vulnerabilidade e bem-estar psicológicos do estudante universitário. Revista Brasileira de Terapias Cognitivas, 10(1), 02-10. https://dx.doi.org/10.5935/1808-5687.20140002

Pinto, F. N. F. R., Barham, E. J., \& Del Prette, Z. A. P. (2016). Interpersonal conflicts among family caregivers of the elderly: The importance of social skills. Paidéia (Ribeirão Preto), 26(64), 161-170. doi:10.1590/198243272664201605

Pureza, J., R., Rusch, S. G. de S., Wagner, M., \& Oliveira, M. da S. (2012). Social Skills Training for College Students: a intervention proposal. Revista Brasileira de Terapias Cognitivas, 8(1), 2-9. https://doi.org/10.5935/18085687.20120002

Andretta, I., \& Oliveira, M. S., (2011) Modelo cognitivo-comportamental do transtorno de ansiedade generalizada. In Andretta, I., Bortolon, C., \& Zambom, L. F., Manual Prático da Terapia Cognitivo Comportamental (pp 289301). São Paulo. Sp. Casa do Psicólogo.

Ribeiro, D. C., \& Bolsoni-Silva, A. T. (2011). Potencialidades e dificuldades interpessoais de universitários: estudo de caracterização. Acta Comportamentalia, 19(2), 205-224. Recuperado de http://pepsic.bvsalud.org/scielo. php?script $=$ sci_arttext\&pid $=$ S0188-81452011000200005\&lng $=$ pt\&tlng $=$ pt.

Santos, R. M. (2014) Perfil de ansiedade em estudantes universitários de curso da área da saúde. Dissertação de mestrado, Universidade Estadual de Paraíba, Campina Grande.

Silva, A. T. B., Leme, V.B.R., Lima A. M. B. L. M, Costa, F.M.,\& Correia, M.R.G. (2009). Avaliação de um Treinamento de Habilidades Sociais (THS) com Universitários e Recém-Formados. Interação em Psicologia, 13(2), 241-251. Recuperado de http://revistas.ufpr.br/psicologia/article/view/13597/11370.

Submetido em: 8-1-2018

Aceito em: 18-12-2018 